ÉGYPTE

monde arabe

\section{Égypte/Monde arabe}

23 | 1995

Géographies de l'Égypte 2

\title{
Aménagement de la ville de Port-Saïd, le point de vue de l'architecte
}

Image idéale, méthodes et déconstruction du rêve

\section{Frédérique Bruyas}

\section{(2) OpenEdition}

\section{Journals}

Édition électronique

URL : https://journals.openedition.org/ema/969

DOI : 10.4000/ema.969

ISSN : 2090-7273

Éditeur

CEDEJ - Centre d'études et de documentation économiques juridiques et sociales

Édition imprimée

Date de publication : 30 septembre 1995

Pagination : 131-168

ISSN : 1110-5097

Référence électronique

Frédérique Bruyas, «Aménagement de la ville de Port-Saïd, le point de vue de l'architecte », Égypte/ Monde arabe [En ligne], 23 | 1995, mis en ligne le 08 juillet 2008, consulté le 07 juillet 2022. URL : http:// journals.openedition.org/ema/969; DOI : https://doi.org/10.4000/ema.969

Ce document a été généré automatiquement le 7 juillet 2022

Tous droits réservés 


\section{Aménagement de la ville de Port- Saiid, le point de vue de l'architecte}

Image idéale, méthodes et déconstruction du rêve

\section{Frédérique Bruyas}

1 En 1973, avec la fin de la guerre israélo-égyptienne, le gouvernement égyptien commence à s'intéresser aux régions périphériques, notamment celle du canal de Suez paralysée par six années de guerre froide. Une vaste opération d'aménagement régional et urbain accompagne la reconstruction, faisant appel à la participation d'experts étrangers et à la planification par schémas directeurs. L'opportunité et l'efficacité de ce type de méthodes sont souvent mises en question devant l'écart entre les objectifs et les réalisations. Aussi avons-nous tenté, à partir du cas de l'aménagement de la ville de Port-Saïd - située à l'entrée méditerranéenne du canal de Suez (cf. figure 1 : localisation de Port-Saïd) -, de cerner la nature et la fonction d'un processus d'aménagement.

2 Nous avons choisi d'analyser en priorité le rôle de cette politique et le discours qui lui est associé. Cette approche, complétée par une étude des documents préparatoires et des déclarations et enjeux politiques, pourrait permettre de comprendre la distribution des responsabilités et des influences qui interfèrent avec le projet à différents niveaux. 
Figure 1 : Localisation de Port-Saïd

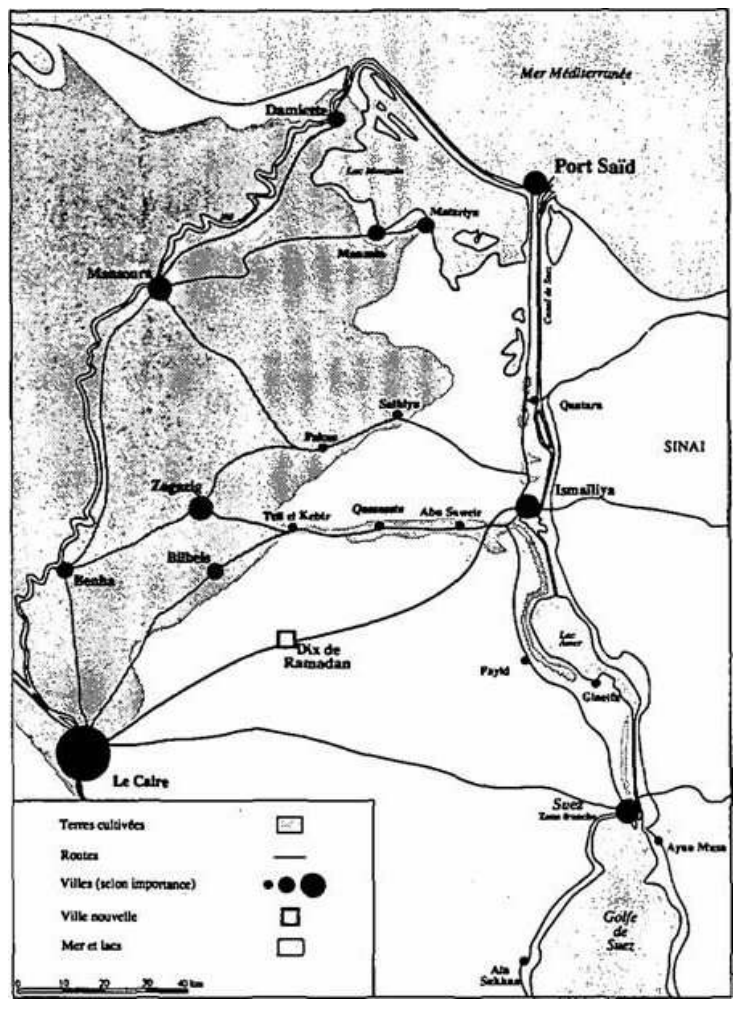

3 Il nous a paru judicieux d'interroger un acteur sur son rôle depuis l'origine du projet jusqu'à sa mise en œuvre, ainsi que sur le déroulement des opérations et le sens qu'a eue pour lui chaque étape. C'est autour du témoignage de Yahya al-Zayni, un des architectes-urbanistes ayant participé à la reconstruction et à l'aménagement de PortSaïd, que nous avons retranscrit les temps forts d'un processus d'aménagement. C'est son discours de témoin et d'acteur qui nous intéresse essentiellement ici. Lors d'entretiens non directifs sur la conception et l'application du schéma directeur de Port-Saïd, depuis les origines du projet (1973) jusqu'à l'approbation du document d'aménagement (1976) et son application, il nous a fait part de son interprétation des méthodes et enjeux de l'aménagement de Port-Saïd, dont la particularité est d'avoir débuté au moment même de la reconstruction. A travers sa vision d'aménageurarchitecte, nous abordons la question de l'aménagement urbain, ses modèles, sa finalité, sous l'angle qu'en donne un acteur directement impliqué.

Diplômé d'architecture de l'École des Beaux Arts du Caire puis de Paris, Yahya al-Zayni est un des architectes qui ont suivi au quotidien la reconstruction de Port-Saïd et l'élaboration du projet de schéma directeur. Il a ensuite dirigé, jusqu'en 1981, le bureau arabe d'études techniques chargé de l'exécution de ce projet. Il a alors été nommé conseiller au ministère du Développement, puis au ministère des Affaires étrangères pour prendre en charge les constructions réalisées outre-mer par l'Égypte.

5 Si l'expérience de Yahia al-Zayni est précieuse pour comprendre le déroulement des opérations, le rôle des différents responsables et la prise de décision, elle lui permet en outre de proposer une réflexion globale sur l'aménagement, ses méthodes et ses enjeux. Son discours semble significatif d'une "manière de faire » - pas forcément spécifique à l'Égypte - doublée d'un regard a posteriori, nourri par de nombreuses années d'expérience dans le domaine du conseil en aménagement. Ces considérations, 
ajoutées au récit du processus d'aménagement et de reconstruction de Port-Saï, nous ont permis de dégager certains points qui nous intéressaient particulièrement. Nous les avons confrontées au discours qui prévaut actuellement sur la scène politique égyptienne, à travers les documents d'aménagement et certains ouvrages conceptuels.

Dans les propos de Yahia al-Zayni', nous avons d'abord remarqué l'importance de la part accordée au grand rêve de transformation d'une ville dévastée en un pôle de croissance d'avenir. C'est par ce biais que nous aborderons la question de l'aménagement avant de tenter, grâce à une analyse des méthodes employées, de cerner par quels mécanismes ce rêve fut déconstruit, et de nous pencher plus spécifiquement sur quelques procédés d'aménagement et d'urbanisme qui se prêtent, d'après l'expérience de Port-Saïd et selon l'avis de Yahya al-Zayni, à une remise en cause.

Conditions d'élaboration du schéma directeur

7 L'aménagement de Port-Saïd est indissociable du contexte de l'après-guerre et de l'intérêt stratégique que présente toute la zone du canal. Depuis son origine, le développement $\mathrm{du}$ port d'entrée méditerranéen du canal de Suez dépend de paramètres propres à la création et au rôle de cette voie de navigation intercontinentale. Comme Ismaïlia et la nouvelle Suez, ses sœurs du canal, la ville de Port-Saïd fut fondée au cours de la décennie 1855-1865 à l'initiative des ingénieurs français de la Compagnie universelle du canal de Suez, pour être une enclave européenne et un port au service de la navigation et du commerce entre l'Occident et l'Orient. La maitrise du canal et son corollaire, celle du commerce avec l'Orient et du pétrole, provoquera des guerres en plusieurs occasions, brisant à chaque reprise l'essor des villes. Après la nationalisation de la voie d'eau et la crise de Suez en 1956, qui provoque le départ des Européens du canal, Port-Saïd, Ismaïlia et Suez sont plus fortement intégrées au territoire égyptien, mais pour une courte durée : en 1967, les troupes israéliennes occupent le Sinaï juste à l'est du canal. Survient alors une guerre d'usure qui ne cessera qu'après l'assaut de 1973.

8 Alors seulement, la paix qui s'est engagée avec Israël permet aux Égyptiens de prendre en main l'aménagement d'une région nouvelle autour du canal de Suez, avec le soutien de l'Onu.

Le contexte de l'aménagement du territoire et la politique nationale

9 Les conditions de la croissance démographique observée dans la capitale et la vallée du Nil vont intégrer l'aménagement des villes du canal dans l'idée d'une stratégie nationale qui sera seulement explicitée en 1981, mais qui visait déjà à rééquilibrer la répartition de la croissance urbaine en Égypte - la capitale et Alexandrie réunissaient $56 \%$ de la population urbaine en $1976^{2}$.

10 Cet aménagement intervient dans une période charnière de mutation politique - les années soixante-dix - qui voit la fin du conflit israélo-égyptien et la mise en place de la politique d'ouverture (infitâh) du président Sadate. Cette politique plus libérale optant pour l'ouverture aux investisseurs extérieurs ne sera pas sans effet sur la conception d'un modèle de développement urbain transcrit dans l'outil schéma directeur. La zone du canal et ses trois villes constitueront le terrain d'application privilégié de l'infitâh, notamment en raison de ce que pouvait laisser espérer la proximité d'un flux commercial international.

Enjeux et stratégies 
11 Les stratégies nationales sont à l'origine de la priorité accordée à l'aménagement de la zone du canal. L'intérêt porté au canal est évidemment lié au caractère stratégique et à l'intérêt économique de la voie de navigation maritime qui constituait une rente de première importance pour l'Égypte. D'un point de vue territorial, la reconquête du canal laissait présager celle de la péninsule du Sinaï, d'où les troupes israéliennes ne furent totalement évacuées qu'en 1982. Les efforts de paix avec Israël pouvaient accélérer d'autant ce retrait. Par ailleurs, pour Anouar al-Sadate, l'espace vide des déserts était dangereux pour la paix. Aussi fut-il recommandé, dans le schéma directeur de Port-Saïd, de lier plus étroitement les villes du canal avec le Delta et le Sinaï « dans le but d'améliorer les défenses de l'Égypte vers l'est » :

«Ces enjeux orientaient toute la politique régionale. La reconstruction représentait un défi politique pour Sadate. Il misait sur Port-Saïd et les autres villes du canal. Il voulait les reconstruire le plus vite possible pour signifier aux Occidentaux - et surtout aux Américains, qui soutenaient Israël - qu'il entendait parier sur la paix. Par là même. il défiait les Israéliens de s'engager dans une paix durable. C'est ainsi que Sadate a pu obtenir le soutien de Carter et des Occidentaux (Américains, Anglais, Français...), qui ont participé à la reconstruction du canal de Suez et l'ont suivie de très près. »

Le projet d'aménagement ne sert pas seulement une stratégie nationale, il est aussi investi d'une fonction politique internationale. Les participations occidentales à la reconstruction recouvrent à la fois des intérêts économiques et des engagements diplomatiques. Ce n'est pas seulement pour l'Égypte que le canal de Suez constitue une route maritime très importante; c'est aussi pour le transport maritime mondial, puisqu'il permet un gain de $42 \%$ sur la distance de Londres à Bombay.

«C'est pour cette raison qu'après la guerre de 1973, beaucoup de pays s'intéressaient de près au déminage du canal et souhaitaient sa rapide réouverture. C'est dans ce but, et pour des intérêts économiques, que tant d'étrangers soutenaient la reconstruction. »

La voie d'eau représentait, avant la guerre de 1967, la troisième source égyptienne de devises. Cet intérêt pour le canal permettait par ailleurs à l'Égypte de bénéficier de l'aide technique et financière de différents pays occidentaux, notamment des ÉtatsUnis, pour la reconstruction des villes du canal :

«Le premier noyau de l'équipe chargée de prévoir le développement de Port-Saïd était composé d'un groupe d'experts égyptiens et anglais, regroupés par le ministère de la Reconstruction sous l'égide du Programme des Nations Unies pour le Développement, qui veillait aussi à la reconstruction de toute la zone du canal. »

Dans ce contexte, la volonté d'assurer la paix contenait déjà les germes du grand rêve qui fut à l'origine des projets d'aménagement de Port-Saïd.

Le grand rêve d'extension : méthodes d'élaboration du projet, référents et discours

Désertée depuis la guerre de 1967 et évacuée en 1969, Port-Saïd est devenue, en 1973, une "ville fantôme". Si la ville est surtout marquée par la dégradation due à son abandon, les dommages de guerre sont aussi conséquents: "Ce sont surtout les installations portuaires et les routes d'accès qui avaient été visées, mais l'habitat aussi était touché, et des îlots entiers détruits. " L'aménagement sera donc conçu parallèlement aux opérations de reconstruction et en coopération étroite avec des experts étrangers. L'aménagement de toute la zone du canal, et notamment de Port-Saïd, prend dès l'origine l'envergure d'un grand projet de reconstruction nationale.

La démarche de planification du schéma directeur 
16 La démarche suivie pour la planification de Port-Saïd suit théoriquement un schéma que l'on pourrait simplifier ainsi : on fixe un objectif - l'image idéale de cette ville dans le futur -, on utilise la «science" - prospective, techniques d'aménagement et d'urbanisme - pour élaborer le plan qui permettra d'atteindre ces objectifs, puis on applique le plan. L'image de ce que sera la ville dans vingt ans devient le plan, même si ce schéma idéal va résister difficilement à l'épreuve des faits.

17 L'image d'un développement urbain se nourrit du grand changement politique amorcé parAnouar al-Sadate. Le schéma directeur rappelle quelques principes énoncés par ce dernier dans les Documents d'Octobre 1973 ; ils sont importants pour l'élaboration d'un modèle de développement en ce qu'ils expriment d'utopie sociale. Dans les propositions retenues, l'accent est mis sur la «science ", le "progrès » et le «futur »; il s'agit de concevoir :

- « un aménagement pour l'an 2000, destiné à fournir les moyens d'un progrès continu pour les futures générations ;

- un aménagement global et efficace pour assurer, par la science, la réalisation des grands objectifs de la société ;

- le développement social et l'amélioration de la condition humaine ;

- le développement culturel basé sur la science et la foi ;

- une société sûre pour les citoyens du présent et du futur ;

- l'entrée dans l'ère de la science et de la technologie. » D'autres éléments sont associés à cette projection d'une société idéale en vue de définir des objectifs de développement. Eléments du contexte historique et géographique

Dans les années soixante-dix, il devient nécessaire de fournir un exutoire à la croissance démographique concentrée dans l'Égypte nilotique et notamment au Caire. Toute la stratégie d'aménagement régional dans laquelle s'insère l'aménagement urbain de Port-Saïd est conçue comme une réponse à une croissance démographique intense, continue et jugée inéluctable. Son objectif premier : planifier l'accroissement de la ville pour lui permettre d'accueillir 800.000 habitants en l'an 2000.

Les options choisies pour Port-Saïd se fondent sur des réalités locales, notamment la référence aux activités passées de la ville. Les ingénieurs français de la Compagnie universelle du canal de Suez avaient en effet fondé et développé une cité portuaire vouée au service des navires empruntant la voie d'eau :

« Nous étions chargés d'étudier les activités récentes du port et de nous en inspirer pour déterminer les options à encourager. Depuis sa fondation, les habitants avaient toujours été dépendants du transport maritime. C'est pourquoi nous avons choisi de renforcer la fonction portuaire en créant un autre port, plus important, au sud de l'ancien, desservi par le canal, et d'encourager les industries liées au port, comme la construction navale et les industries de transformation ou d'assemblage qui n'exigent pas de grandes usines mais produisent pour l'exportation, à partir de matières premières importées. Le développement de cette branche devait être favorisé par l'installation de zones franches. Les secteurs privilégiés étaient donc le port, les zones franches, l'industrie maritime et, dans une moindre mesure, le tourisme et la pêche. »

Les contraintes du site sont prises en compte sans pour autant demeurer un obstacle infranchissable :

«Limitée par les eaux de tous côtés, la surface de Pon-Saïd est très restreinte :au nord, la Méditerranée ; à l'est, le canal ; à l'ouest et au sud-ouest, le lac Manzala et, de l'autre côté du canal, le lac Mahalla. Les nouvelles options devaient tenir compte de cette existence maritime, d'une part, parce qu'elle avait donné à la ville sa 
fonction portuaire et, d'autre part, parce que dès les premières étapes, l'aménagement spatial de Port-Saïd dépendait des contraintes naturelles du site. Nous avions prévu de développer la surface construite vers le sud-ouest pour les zones résidentielles, et $\mathrm{du}$ nord au sud du canal pour les activités portuaires, l'industrie et l'entreposage. Il fallait donc penser à gagner des terres sur les eaux du lac Manzala pour établir une zone franche et construire de nouveaux quartiers, puisqu'il était prévu de faire des villes du canal des pôles de croissance. »

Figure 2 : Plan de développement à long terme de Port-Saïd sur le lac Manzala

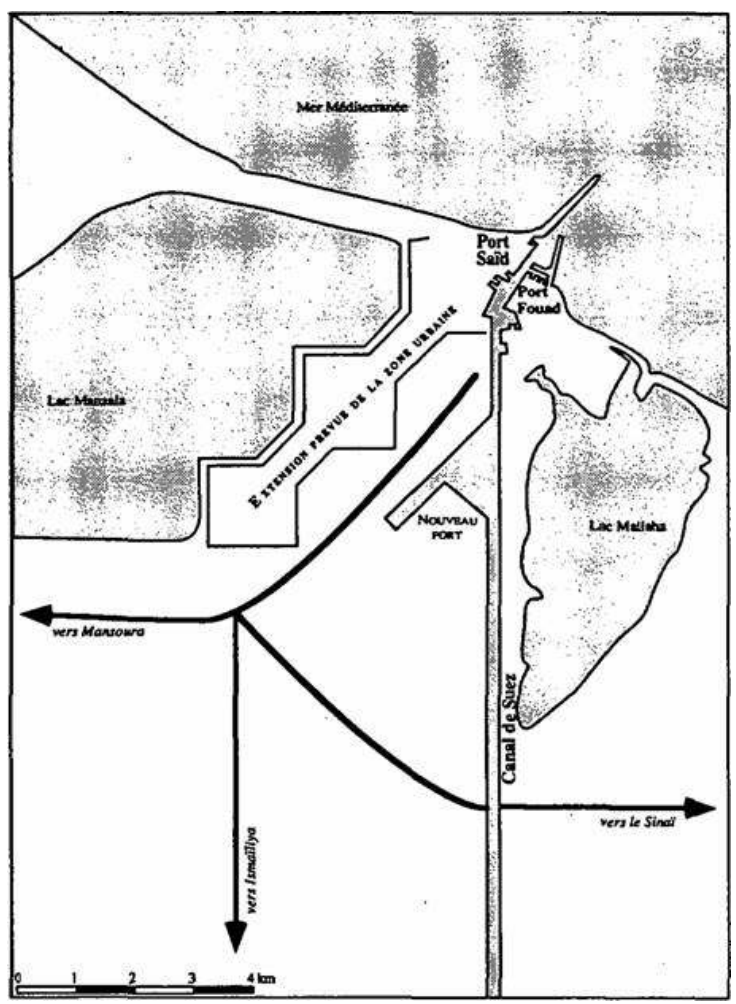

On prévoit alors de gagner 2.370 hectares sur le lac Manzala d'ici l'an 2000, dont 1.525 hectares pour le développement urbain, les 845 restant étant consacrés aux corridors de transport et à l'agrandissement du port et de ses dépendances (cf. figure 2 : plan de développement à long terme de Port-Saïd sur le lac Manzala) :

«Une surface inouie était concernée par ces dragages. Mais pour accueillir toute la population prévue, il ne suffisait pas d'augmenter les densités, qui étaient déjà élevées; il fallait s'étendre sur de nouvelles terres. Nous étions donc obligés de tenir compte de l'environnement. »

Cela signifiait alors développer la ville sur des terrains artificiellement gagnés sur le lac sans mettre en avant des limites de faisabilité ou de coût. Une étude sur la bonification de terres à usage urbain à Port-Saïd fut cependant réalisée à la fin des années soixantedix avec l'assistance technique et financière du Canada. Or, le problème de la pollution $\mathrm{du}$ lac Manzala se pose aujourd'hui pour différentes raisons, dont la principale est le déversement dans le lac des effluents de six gouvernerais. Certains détracteurs profitent de ce problème pour remettre en cause le système de dragage ${ }^{3}$ qui avait été adopté pour gagner de nouvelles terres, ainsi que toute cette stratégie d'extension qui porterait préjudice à la pêche dans le lac.

D'autres options d'aménagement découlèrent du site presque insulaire de Port-Saïd. 
Raccrocher la ville à l'Égypte nilotique

Depuis son origine - elle était alors un camp sommaire sur le cordon littoral entre lac et mer -, la ville de Port-Saïd s'est développée en gagnant des terres sur l'élément liquide qui la cernait, comme sortie des eaux. Aussi les axes de développement se devaient-ils de rattacher la cité à la vallée du Nil, artère d'un territoire centralisé, puisque par ailleurs Port-Saïd était déjà reliée à Isma'ilia et Suez par le canal et les routes qui le longent.

«Alors, il fallait chercher d'autres liens avec la terre-mère, c'est-à-dire le delta.

C'est pourquoi nous avons proposé un axe secondaire reliant la ville à Mansoura,

vers l'ouest. »

L'expression "terre-mère " paraît exprimer assez clairement la question, qui se posait alors, de la réappropriation d'un espace en marge. Cette perspective vaut pour toutes les villes du canal, mais le cas de Port-Saïd est plus éloquent, car on peut considérer la ville comme née de la mer ou du canal de Suez. Si une administration égyptienne a été mise en place peu après sa fondation, la ville est née dans les limites d'une concession accordée à la Compagnie universelle du canal de Suez, dont le pouvoir dans la gestion de l'espace a été prépondérant jusqu'en 1956, alors que la surface urbaine s'étendait bien au-delà de ces limites. La zone du canal représentait le dernier front d'urbanisation vers l'est; arrière-garde militaire de l'Angleterre avant 1956, elle est devenue une zone de combats à plusieurs reprises à partir de 1948. Entre la nationalisation du canal en 1956 et la guerre de 1967, l'Égypte, libérée de la présence de la Compagnie universelle, pouvait orienter plus librement l'évolution du canal et de ses villes, mais ce n'est qu'après 1973, lorsque la paix prend véritablement forme autour du canal, que toute la zone - dont Port-Saïd - peut enfin être rattachée plus étroitement au reste du pays.

Pourquoi renforcer absolument les liens de Port-Saïd avec le delta si, en dehors de l'approvisionnement en produits agricoles, la ville tire de la mer l'essentiel de ses activités (pèche, industries d'importation-exportation...) ? Précisons que les conditions historiques avaient valu à la population la réputation d'être indépendante, voire rebelle à toute forme d'autorité, privilégiant l'autonomie traditionnelle d'un peuple de marchands, de marins et de grands commerçants qui se perpétuera avec la zone franche. Dans ce cadre, des liaisons plus fortes avec le delta s'avéraient nécessaires pour que l'économie du pays tire des bénéfices de l'activité marchande de Port-Saïd. La «terre-mère » entend bien remettre la main sur cette cité en marge, trop soucieuse de son indépendance :

«On ne pouvait pas se contenter d'une économie de transit pour développer la

ville, Port-Saïd devait devenir un pôle de croissance économique pour tout le

pays. »

Dans ce but, le port devait passer d'une fonction d'escale, de transit «extérieure à l'Égypte» (à l'exception de l'importation de certains produits venant d'Asie), à une fonction de transit fructueuse à l'échelle nationale : l'Égypte importe des marchandises qui doivent entrer dans le pays, d'autres sont à exporter directement, certaines à mettre en dépôt quelque temps en zone franche, puis à réexporter.. Autre moyen de renvoyer les bénéfices de son développement à l'Égypte; renforcer sa fonction d'approvisionnement pour tout le pays. 
28 La nécessité de raccorder étroitement la zone du canal au reste du paye et d'y inscrire plus fortement la présence de l'État central ont contribué à formuler de manière volontariste le grand projet d'aménagement.

Reconstruction d'une ville : tout est possible...

«Nous nous laissions parfois emporter par les objectifs de croissance: dans la première étude, le chef de projet pensait multiplier par trois ou quatre la surface qui était celle de Port-Saïd en 1973, en direction du sud-ouest. C'était vraiment trop ambitieux, mais nous rêvions de faire des choses exceptionnelles. C'était avant tout les rêves des Égyptiens et des membres du groupe de planification qui travaillaient sur le projet, et comme il n'y avait pas d'orientation de la part du gouvernement à cette époque, nous avions toute liberté d'imaginer ce qui nous plaisait, sans contraintes. Nous voyions les choses en grand parce que ces projets correspondaient à des études tout à fait préliminaires et qu'à ce moment-là, nous n'étions pas contraints par les conditions réelles, qu'elles soient économiques ou sociales. Ces projets étaient d'ailleurs adaptés aux prévisions, puisque nous avions estimé que la population de Port-Saïd devait atteindre près de 800.000 habitants en l'an 2000.»

Les éléments d'utopie du projet urbain

29 L'ambiance de la reconstruction, d'abord, favorise la conception de projets porteurs d'idéaux nouveaux : la ville a été désertée depuis quatre ans, rien ne subsiste si ce n'est quelques éléments de structure, mais toute l'activité est à relancer. On peut repartir sur de nouvelles bases, tout inventer, créer une société nouvelle. Ce dernier objectif se trouve être aussi au cœur de la politique du président Sadate, qui prône «un aménagement global et efficace pour assurer, par la science, la réalisation des grands objectifs de la société, un développement social et l'amélioration de la condition humaine " [Documents d'Octobre 1973). A Port-Saïd, tout est à rebâtir; comme les autres villes du canal, elle peut constituer le creuset de cette «nouvelle société ».

30 Car si la préoccupation prioritaire des aménageurs concerne l'extension périphérique, tout le projet urbain sous-tend des éléments d'utopie sociale. Cela se traduit notamment par la volonté de fournir harmonieusement les équipements et services nécessaires à chaque catégorie et quartier.

31 Peut-on pour autant affirmer que « la ville apparaît à la fois comme un point fort du projet social et comme une métaphore (...) de ce projet» (J. Lévy, 1994, p. 325), sans exagérer l'importance accordée sciemment à la ville dans le projet de développement général ? Disons plutôt que si cette nouvelle société n'est pas spatialisée dans les projets d'Anouar al-Sadate, elle trouve naturellement son accomplissement dans les villes du canal puisqu'il s'agit alors d'une "re-création ». Le projet urbain et le projet de société sont associés.

Le thème-clé du créateur-démiurge

32 Les aménageurs qui envisagent de tout reconstruire en tenant compte d'un idéal social s'apparentent en partie au créateur qui dose savamment chaque composante de sa ville. Sur les plans, tout est possible. Comme pour réaliser une ville nouvelle sur un site vierge, on prévoit à Port-Saïd de créer de l'espace au-delà des éléments, on trace à coups de crayon une extension qui défie les contraintes naturelles :

"Quand on commence un projet comme celui-ci, tous les chercheurs, aménageurs

et architectes voient les choses en grand. Parfois, en outre, ils ne sont pas limités par la réalité. C'est ce qui s'est passé pour Port-Saïd. »

33 Le volontarisme entretenu par les autorités centrales permet aux aménageurs qui en héritent de croire à cette ré-création. Ici, le démiurge qui trace l'image de ce que sera la 
ville 25 ans plus tard s'oppose à «l'individu pleinement conscient des limites de toute attitude volontariste, non seulement vis-à-vis des intérêts privés mais aussi des interventions de l'État et de ses grandes institutions" (C. Chaline, 1989). La mobilisation internationale, le chantier national, tout concourt à laisser émerger les idées les plus prometteuses.

L'image de la ville : entre modernité et originalité

Au départ, les planificateurs ne formulent pas une idée de la ville à proprement parler, mais envisagent le développement d'une image préexistante. En revanche, leurs projets et, la manière dont ils les expriment, associés au renouveau politique du moment, nous laissent découvrir une image projetée, nourrie à la fois des spécificités locales et des influences qui ont formé les aménageurs. Ainsi, au début des recherches sur le développement de la ville, l'équipe anglaise avait eu l'idée de profiter du caractère pratiquement insulaire de Port-Saïd pour remodeler la ville autour de canaux et encourager la navigation par voie fluviale, sur le modèle de la trame urbaine de Venise :

«Selon eux, cela pouvait permettre de se passer d'automobiles et de minimiser la pollution. Celui qui avait eu cette idée avait pris cela très au sérieux : il pensait avoir trouvé un concept qui pouvait rendre la ville très intéressante. Des plans ont même été conçus, mais nous n'étions pas d'accord. »

Dans la recherche d'une image attractive pour Port-Saïd, ce type d'originalité parut coûteux et peu en accord avec les nécessités du moment. Trouver un moyen de la hisser au rang de ville moderne représentait un espoir plus crédible et une image plus immédiatement rentable pour attirer les emplois et la population qu'ils étaient susceptibles de drainer. Le canal de Suez, cet ouvrage exceptionnel, n'avait-il pas été un temps porteur de modernité et d'activités nouvelles (dès 1902, une zone franche avait été créée et jusqu'en 1956, le port était classé parmi les premiers du monde) ? En 1973, le canal et la rente qu'il constitue sont à nouveau disponibles. Aussi, avec en arrièrepensée l'image de Singapour dont le port et la zone franche avaient bien fonctionné à partir de 1965, le statut de ville franche est accordé à Port-Saïd le 7 mai 1975. Parmi les autres signes d'une volonté de modernisation, toujours d'actualité dans les années quatre-vingt-dix pour la zone du canal, notons la construction de la wâdi al-technologia (" vallée de la haute technologie ») au nord d'Ismaïlia, sur la rive est du canal.

Il ne faut pas négliger la portée de cette image affichée de modernité, mais c'est finalement sur la base de l'industrialisation et, surtout, sur le développement commercial que se fera la croissance urbaine. Selon E. Longuenesse (1990), il importe de garder en mémoire la spécificité de tout projet politique dans un pays en voie de développement, et la force d'aspiration à la modernisation.

Aussi les objectifs des projets du schéma directeur de Port-Saïd sont-ils spécifiquement conçus pour servir l'image de la ville. L'exemple le plus significatif est l'option du gain de terres sur les eaux du lac Manzala, qui suggère bien le recours à des techniques modernes et constitue un argument imparable contre certains projets, jugés moins porteurs en terme d'image publique :

\footnotetext{
«Le projet de reconstruction en dur du quartier de cabanes Qabutti ${ }^{4}$ avec des maisons très fonctionnelles et des ateliers pour les bateaux des pêcheurs a été refusé parce qu'on nous a opposé le fait qu'il fallait en priorité gagner des terres sur le lac Manzala. Pourtant, il était entendu qu'à l'époque, la reconstruction devait passer avant la bonification. »
} arrivée. Les objectifs visaient davantage à attirer de nouveaux résidants ou à répondre 
à des enjeux politiques et économiques qu'à subvenir aux besoins des citoyens en place. Les objectifs, les choix de la politique d'aménagement sont destinés à servir l'image, à se rapprocher de sa matérialisation. L'image projetée est un modèle officiel dans lequel réside l'espoir de mettre en place des solutions à la croissance et de voir disparaitre les problèmes qui se posent. C'est un «modèle territorial de représentations" qui est explicité dans ce projet, « celui qui s'affirme en tant qu'officiel. Comme tel, il prétend s'imposer à l'ensemble du corps social. Il ressortit à l'idéologie territoriale dominante. » (M. Lussault, 1993, p. 289)

L'image a une fonction. Dans ce cas précis, c'est d'être diffusée au travers d'un discours afin de servir les enjeux en question. En rendant publique une certaine image de PortSaïd, on espère toucher ceux que l'on souhaite attirer dans ce nouveau pôle de croissance. Selon Yahya al-Zayni, « il s'agit davantage de discours que de vues réalistes ».

La communication : instrument de l'action

L'image accolée au projet de Port-Saïd est diffusée par un discours qui s'exprime sur le mode volontariste et traduit l'optimisme en jeu à ce moment de la planification :

«Ces études étaient optimistes parce qu'alors, on ne connaissait pas de limites et on voyait grand, quoiqu'il arrivait on devait voir grand!»

41 Si le discours est investi de différentes fonctions, il doit d'abord permettre d'atteindre les objectifs fixés. Avant toute chose, l'image idéale de Port-Saïd et du canal, projetée dans le discours, a pour objectif de mobiliser la population autour de la reconstruction :

« $\mathrm{Au}$ moment où nous étions en train de formuler toutes sortes d'idées dans les rapports commandités, c'était vraiment la 'bataille de fa reconstruction', c'était d'ailleurs devenu un slogan... »

42 C'est un vocable appartenant au registre guerrier qui illustre la mobilisation à laquelle les pouvoirs publics tenaient particulièrement pour encourager le processus de paix. Le discours a un rôle stratégique. Proclamer les efforts accomplis pour développer la ville que ses habitants qualifient parfois de "martyre » en rapport avec toutes les guerres subies, traduit aussi la préoccupation de maintenir la paix sociale par l'affirmation de projets tendant au bien-être général.

Mais le discours n'est pas seulement prononcé à destination de la population égyptienne, il vise aussi les politiques et les investisseurs internationaux. Conçu comme un argument publicitaire et stratégique, il est alors investi d'enjeux politiques et économiques. Sur ce plan, on fait toujours confiance à l'idée de l'intégration à la sphère internationale, objet de tous les espoirs et convoitises. "Aujourd'hui, ce souci d'internationalisation semble souvent premier, car plus médiatique que la simple recherche d'activité dans la mesure où il permet de déployer une véritable stratégie d'image territoriale forte.» (M. Lussault, 1993, p. 23) Décrivant à l'origine la stratégie d'image de la ville de Tours, ces mots illustrent bien que la communication d'une image forte et attractive compte avant tout. C'est dans ce but, pour attirer la population et les investisseurs, que l'on a aussi recours au registre de l'internationalisation et à l'idée de progrès; c'est toujours dans ce but que le discours est diffusé sur le mode de l'optimisme.

Déconcentrer la vallée pour occuper des territoires ciblés

Ces projets et le discours qui les sous-tend s'inscrivent dans le contexte de la politique régionale égyptienne. Ils nous amènent à réfléchir sur la manière dont les aménageurs et le pouvoir central, qui leur propose d'atteindre des objectifs démographiques donnés, envisagent la mobilité des populations et la répartition des investissements. A 
partir des années soixante-dix, la distribution de la croissance urbaine sur tout le territoire égyptien est à l'origine d'une politique urbaine nationale formalisée en 1981 dans le National Urban Policy Study. Il a pour objectif d'attirer la population des régions surpeuplées vers des points d'attraction éloignés de la vallée du Nil afin de réduire les densités de la vallée en commençant par peupler les oasis, le canal de Suez puis le Sinaï. Il est prévu que les villes du canal absorberont une bonne part de cette croissance.

Comme le résume C. Chaline, la planification nationale et régionale en Égypte n'est pas dépourvue de contradictions. Alors qu'apparaît l'idée de prescrire un report géographique de la croissance urbaine vers les régions encore peu développées de Suez et du Sinaï, l'État n'échappe pas à la tentation de conforter la croissance métropolitaine du Caire en la redistribuant dans des villes satellites à la périphérie. Dans un cas comme dans l'autre, on imagine pouvoir diriger la population vers les sites choisis : mais si pour les villes satellites du Caire des relogements autoritaires ont permis d'amorcer les migrations, seule la prospérité pourrait attirer la population vers les villes du canal. En réorganisant la répartition de la population sur le territoire, les planificateurs et dirigeants trahissent parfois leur intention de disposer des gens et de l'espace. Fixer le nombre des habitants d'une ville, c'est présupposer de la volonté des individus à migrer. A Port-Saïd, c'est aussi miser sur le peuplement d'une zone tampon, immédiatement après la guerre, pour la remettre rapidement à distance des canons israéliens.

Cela nécessite de sérieuses incitations. Ainsi, l'idée de redonner à Port-Saïd un rang international grâce à la zone franche et au développement du tourisme avec un nouvel aéroport international apparaît comme la solution idéale :

«La meilleure façon d'attirer la population, c'est de créer des emplois. Dans le schéma directeur, nous mettions surtout l'accent sur le développement d'une zone franche de très grande importance. On pariait de faire un nouveau Singapour. Le développement de Port-Saïd n'a pas fonctionné aussi vite, ni si bien, mais au moment où nous rêvions de ce projet pilote, c'était vraiment dans l'espoir qu'il atteigne ces objectifs le plus vite possible.»

47 Cependant, le chiffre de population visé n'a pas été atteint alors qu'on offrait à celle-ci une "ville de rêve ». Malgré le repeuplement rapide de Port-Saïd à partir de 1974, la ville comptait «seulement» 490.000 habitants en $1990^{5}$. La croissance escomptée ne s'est pas poursuivie selon le rythme prévu:

«Atteindre le million d'habitants aurait nécessité beaucoup plus de temps que les 25 années annoncées dans le schéma directeur, peut-être trois ou quatre fois plus. »

Si les délais annoncés n'ont pas été respectés, retard qui illustre aussi la surestimation des objectifs, il ne s'agit pas de remettre en cause la réussite des projets. Selon les estimations de la Capmas, Port-Saïd est désormais la ville la plus riche d'Égypte avec un revenu moyen de 7.731 fe (livres égyptiennes) par an et par ménage, contre une moyenne nationale de $5.428 \mathrm{fe}$ en 1991. Une certaine part de la population a finalement été attirée par l'espoir d'un profit rapide lié aux activités commerciales de la ville franche. Paradoxalement, ces enrichissements sans réinvestissement dans l'industrie locale ont finalement limité les effets attendus de la mise en place de la ville franche, ce qui explique aussi l'infléchissement relatif de la croissance. 

visent la concrétisation de cet idéal urbain suggèrent d'autres éléments pour l'évaluation de cette politique.

Techniques d'urbanisme et coopération fait appel à la coopération d'experts internationaux. Dans le contexte de la mobilisation internationale pour la reconstruction, on fait d'abord confiance aux sciences de l'aménagement et de l'urbanisme ainsi qu'aux «techniques modernes » des experts étrangers. Les propos de Yahya al-Zayni laissent entendre que cette confiance est relative. Nous verrons plus loin comment s'est posée la question des modèles au sein de l'équipe.

Les moyens d'obtenir l'image idéale : mise en place de la coopération

L'équipe de conception du schéma directeur est pluridisciplinaire, composée d'experts étrangers, de coopérants et du bureau égyptien de conseil dépendant du ministère de la Reconstruction. Yahya al-Zayni insiste sur l'importance du travail d'équipe et du partage des tâches, en précisant toutefois que

«les Anglais étaient plus nombreux parce qu'ils participaient au projet au titre de l'aide et qu'ils étaient payés par leur gouvernement. Nous travaillions dans les mêmes bureaux, nous discutions des options et concevions les projets ensemble. »

Soulignant le rôle des planificateurs égyptiens dans ce projet, il ajoute :

«Le tracé des plans définitifs, du point de vue du dessin uniquement, était le plus souvent le travail d'Égyptiens. »

53 Nous verrons par la suite comment cette remarque peut nous amener à mettre en avant les effets pervers de la coopération. Selon Yahya al-Zayni, il s'agit bien d'une aide extérieure que l'on pourrait qualifier « d'appoint »:

«Il ne s'agissait pas d'une assistance destinée à des aménageurs moins compétents. On ne l'avait pas réclamée pour réaliser des études et des travaux que nous ne savions pas faire. C'était une forme de coopération établie entre les gouvernements. »

L'idée de mobilisation internationale prend forme. Le monde s'associe à l'Égypte par solidarité, mais aussi parce que toute coopération sous-entend un gain de part et d'autre :

"Dès que le président Sadate a pris la décision de récupérer le canal de Suez Jusqu'à l'autre rive du Sinaï, le monde occidental a voulu témoigner sa sympathie en aidant l'Égypte. Cette opération d'entraide a d'abord permis de financer le premier objectif de cette coopération, le déminage et le déblaiement du canal de Suez, auquel les Américains ont pris une grande part. Les Anglais participaient aux travaux à PortSaïd, des coopérants d'autres pays travaillaient dans les autres villes du canal. Après le départ des Anglo-saxons, les Japonais ont coopéré avec l'Organisme du canal. Ils se sont chargés d'approfondir et d'élargir le canal et de réaliser les branches de dérivation du canal maritime. La coopération se déroulait aussi dans l'intérêt des nations qui proposaient leur assistance: le Japon utilisera ensuite l'Égypte comme porte d'entrée des marchés moyen-orientaux. "

De cette «opération d'entraide » dont Yahya al-Zayni se fait l'écho résulte un projet dont les références et les formations sont à dominante anglo-saxonne. Le rôle des experts étrangers, prépondérant lors de la phase de conception du projet, va s'estomper lors de sa mise en œuvre.

Le schéma directeur : un outil « scientifique » pour concevoir la ville rêvée 
Selon Yahia al-Zayni, il n'y avait guère de possibilités de désaccord dans l'élaboration du schéma directeur :

«En ce qui concerne l'idée générale du plan, nous étions d'accord parce que les principes en étaient simples. Il s'agissait d'analyser les possibilités et les contraintes $\mathrm{du}$ lieu. Les critères d'élaboration du schéma directeur font appel à des principes d'urbanisme qui découlent d'une science logique, on ne peut donc pas avoir des points de vue très contradictoires. En fonction des contraintes et de l'emplacement spécifique de Port-Saïd, la viile ne pouvait que s'agrandir vers le sud ou le sudouest. Il n'y avait donc pas beaucoup de variation entre nos idées respectives. »

Toutes les catégories d'experts s'accordent à programmer l'accroissement de la ville sur le lac Manzala ; plus que les potentiels existants - comme l'insularité, idéale pour une zone franche -, la nécessité d'un gain de terres sur les eaux du lac crée un consensus, garantie pour atteindre les objectifs de croissance et de modernisation de Port-Saïd :

«Par contre, sur les projets plus précis qui n'engageaient pas tout le schéma directeur, sur des lieux définis et à l'échelle de l'occupation du sol, nous avions parfois des idées divergentes. »

Car une fois décidé l'accroissement de la surface de Port-Saïd, reste à lotir les nouveaux terrains, à organiser le développement de l'agglomération en continuité avec le tissu existant. Yahya al-Zayni rapporte que la conception de plans plus détaillés fait parfois surgir des divergences entre aménageurs étrangers et égyptiens. Les experts étrangers auraient parfois tendance à oublier certaines des conditions réelles, qu'elles soient locales, financières ou liées au contexte des priorités nationales. L'exemple du projet avorté de transformation de Port-Saïd en "Venise égyptienne», proposé dans le schéma directeur, est significatif de la manière dont les experts étrangers tentent parfois des expérimentations (E. Longuenesse, 1990).

«D'après nous, l'idée [de « Venise égyptienne »] était dangereuse et très onéreuse : les infrastructures élémentaires de la ville étaient dans un état lamentable après les bombardements, et leur capacité était déjà dépassée. Il n'était pas question de creuser des canaux avant de réaliser les infrastructures nécessaires aux besoins immédiats de la population. Nous avons vite abandonné cette idée, mais elle exprime bien la contradiction qui pouvait survenir entre le point du vue, absolument réaliste, de celui qui sent l'urgence de rapatrier tous les immigrés, et celui de l'expert parfois tenté par des fantaisies ou des expérimentations. »

S'il peut comporter quelques éléments de fantaisie résultant des premières réflexions, et dont chacun sait qu'ils ne seront pas appliqués, le schéma directeur apparait avant tout comme un "outil scientifique». Aussi, l'aménagement - celui qui permet de réaliser des documents généraux - serait une science fiable et non remise en cause, qui est partout la même puisque elle est basée sur une analyse «logique » des problèmes. On pourrait avoir confiance en cette science qui, par ses méthodes d'analyse - porter un regard neuf sur les problèmes pour y apporter des réponses -, propose des solutions à toutes sortes de questions (essentiellement axées sur la mise en place d'infrastructures de communication et la répartition spatiale des activités). L'urbanisme, qui concerne l'organisation d'espaces plus restreints (quartier, îlot), souvent sur la base de modèles, et qu'il oppose à l'aménagement généraliste, pose davantage de problèmes. Nous verrons par la suite que les critiques adressées à l'urbanisme pourraient s'appliquer au schéma directeur tout entier.

Remise en cause de la légitimité d'un urbanisme universel 
60 Selon Yahya al-Zayni, l'urbanisme, si on l'entend comme «science ou art de la réalisation de villes ou de quartiers urbains " ${ }^{6}$, dépend des relations sociales et traduit les habitudes de vie d'une société. Dès lors, il est naturel que survienne une confrontation d'idées entre experts égyptiens et étrangers :

«En matière d'urbanisme, lorsqu'on étudie l'emplacement des bâtiments, les surfaces libres entre les éléments du bâti, les relations entre les services et les zones d'habitat, les plans des logements, chacun a des conceptions variées parce qu'elles dépendent des relations sociales. Lorsqu'on a établi les plans de détail, on a constaté que les experts anglais appliquaient des données occidentales, étrangères au pays et à la région. »

61 Cette différence s'illustre dans la conception du plan type d'unité de voisinage qui devait organiser la construction des nouveaux quartiers d'habitat (cf. figure 3 page suivante : plan type d'unité de voisinage). Quatre de ces unités composent un quartier, lequel dispose de services tels que mosquée centrale, écoles secondaires, grand souk... La manière dont Yahya al-Zayni analyse aujourd'hui ces unités soulève d'abord la question de l'adaptation des données de bases à la société pour laquelle est prévu l'aménagement :

«Les experts avaient décrété que l'unité de voisinage devait regrouper 5.000 habitants en 1.000 logements et que les enfants ne devaient pas avoir à parcourir plus de 500 mètres pour se rendre à l'école... L'idée d'installer les services dans le centre des unités de voisinage, voire l'idée d' "unité de voisinage » elle-même, reposait sur le modèle de la société occidentale, dans laquelle la vie de la rue n'est pas très développée. Ce n'est pas le cas ici, où la vie de la rue est très importante et joue un rôle social. "

62 Yahya al-Zayni relève d'autres problèmes, comme l'insuffisance d'une seule mosquée centrale pour un nombre d'habitants aussi élevé, la circulation hiérarchisée et quadrillée mais sans passage piétonnier, ou encore un espace entièrement organisé autour de la grande place centrale. Réagissant aux techniques de l'urbanisme, en particulier à la séparation dans l'espace - à l'échelle du quartier- des fonctions urbaines, il étaye son discours d'une comparaison avec le cas exemplaire de Brasilia dont le centre, " organisé autour d'un grand axe à l'extrémité duquel siégeaient les haut-lieux administratifs, le parlement et les ministères, a été finalement diversifié pour ramener un peu de vie». 
Figure 3 : Le plan type d'unité de voisinage

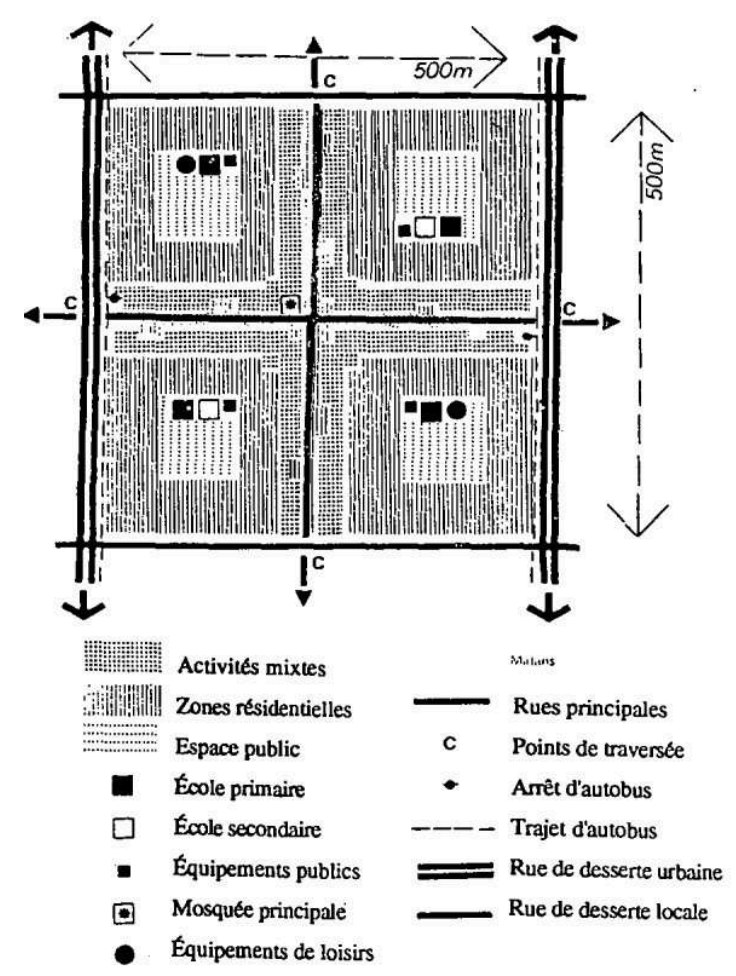

63 Sans pour autant remettre en cause le principe du zonage à l'échelle de la ville, qui a déjà été critiqué parce qu'il représente l'utopie d'un monde parfaitement maîtrisé ou une fonctionnalité exacerbée, il se réfère au zonage de Brasilia pour mettre l'accent sur la nécessité de mélanger les activités dans un quartier résidentiel :

«Ici, nous préférons privilégier les activités mixtes. Même si le centre de l'unité de voisinage regroupe les activités, les artères principales doivent aussi être dotées de commerces et de services. C'est ce que nous avons finalement imposé. »

La séparation des fonctions, lorsqu'elle s'applique à un quartier, est ici critiquée au nom d'un autre mode de vie. C'est donc la validité d'une forme d'urbanisme « universel », d'un modèle applicable en toute situation, qui est en question.

Une critique de l'idée de modèle

L'existence d'un modèle d'urbanisme susceptible de produire le résultat imaginé sousentend l'idée d'un " modèle humain » dont les besoins seraient exactement adaptés à cet urbanisme. Réfléchir à ces questions nous permet de mieux comprendre comment Yahya al-Zayni perçoit le rôle et l'adaptation des modèles à une situation locale.

Un urbanisme fonctionnaliste pour des modèles humains aux besoins identiques

Dans la manière dont Yahia al-Zayni perçoit les unités de développement conçues théoriquement, on peut déceler une remise en cause de ridée de plan type, identique et reproductible à l'infini, suivant les besoins de l'extension périphérique et surtout, valable partout. Elle rejoint une critique souvent formulée à rencontre des urbanistes "progressistes » qui, Le Corbusier en tête, avaient pensé et conçu la ville pour les besoins types d'un homme type (« habiter, travailler, circuler, se cultiver le corps et l'esprit »). Cet urbanisme repose sur une conception a priori de l'individu. M. Roncayolo (1990, p. 168) précise: «La cité idéale de Le Corbusier (...) implique un style de vie 
collectif qui appartient à une société imaginée. » Dans la réalité, les besoins types sont parfois réduits et l'aménagement vise à remplir une fonction en priorité, le logement :

«C'est le plus gros défaut que l'on observe dans les projets de développement. Les responsables croient qu'il est absolument nécessaire de commencer par les logements. Ils les construisent alors à une vitesse folle, sans fournir les services qui encourageraient les gens à venir s'installer. Et c'est seulement quand les habitants se plaignent que l'on commence à répondre à leurs besoins. Cela s'est vu dans plusieurs projets. A Port-Saïd, cela s'explique par les prévisions émises dans le schéma directeur : il fallait donner la priorité à l'habitat pour loger les 798.000 habitants prévus, mais finalement, nous avons opté pour un usage mixte des bâtiments : logements à l'étage, commerces et services en rez-de-chaussée. »

67 Au-delà du fonctionnalisme, il ne faut pas négliger "l'habiter » et les significations multiples de l'établissement humain qui n'apparaissent pas dans les modèles.

68 A partir d'un aménagement que l'on pourrait associer aux modèles progressistes - un urbanisme conçu pour un homme type et un nouveau modèle de société -, Yahya alZayni émet des remarques que l'on pourrait rattacher aux critiques des modèles culturaliste et progressiste, fondées sur une idéologie du même type. Il s'agit de se référer, dans une réflexion sur la ville, non plus à un modèle humain mais à l'homme réel. Les critiques élaborées dans un esprit progressiste préconisaient le recours à des techniques prévisionnelles pour justifier des projets types. Dans cette perspective, Yahya al-Zayni met en avant l'importance de la phase de pronostic. Les critiques se référant aux conceptions des culturalistes proposent de repartir des expériences du passé: l'architecte se réfère au style de vie observé dans des quartiers plus traditionnels. Cela revient aussi à discuter de l'adaptation des modèles.

Adapter un modèle à la société à laquelle il s'appliquera

L'attachement aux valeurs traditionnelles transparaît dans le discours de cet architecte: on y trouve une forme de nostalgie de l'«identité égyptienne» par opposition au mode de vie qu'impose le « rythme de vie moderne ». Il revendique une manière de vivre que l'urbaniste se doit de respecter, prenant comme référence des types d'urbanisation séculaire, souvent non planifiée, comme la vieille ville du Caire. Or, il y a bien longtemps que les programmes d'urbanisme réalisés en Égypte, comme dans d'autres pays soumis à l'influence occidentale, ne reflètent plus l'image que Yahya al-Zayni présente du quartier « oriental». Peut-on en conclure que tout l'urbanisme récent va à rencontre des modes de vie actuels dans les villes égyptiennes? Mieux vaut sans doute observer, avec cet architecte, que le mode de vie traditionnel évolue naturellement dans les quartiers les plus «modernes » du Caire, par exemple ceux qui abritent les fonctions tertiaires de Muhandisîn (quartier construit par et pour les ingénieurs) ou du centre-ville. Par ailleurs, il convient de rappeler que le noyau urbain originel des villes du canal fut conçu sur le modèle occidental de la ville coloniale, avec séparation des quartiers "franc» et «indigène ». Ces villes n'en sont pas moins, aujourd'hui, des villes égyptiennes.

70 A travers l'exemple des plans types, l'architecte témoin pose le problème de l'inadaptation d'un modèle étranger à la société à laquelle il est destiné. Les experts arrivent avec « une idée préconçue de l'urbanisme et sans s'imprégner de la société ».

71 Or, les modèles sont conçus comme des " a priori qui ne traduisent nulle part la réalité concrète d'un lieu», comme des «idéaux détachés de tout préexistant " (F. Choay, 1965, p. 62). N'ayant rien à voir avec la situation locale, doivent-ils lui être adaptés? Pour savoir si l'adaptation de modèles est une nécessité, si elle est légitime, il faudrait 
noter les cas où le besoin s'en est fait sentir, où il s'est traduit par la transformation d'ensembles urbains en vue de les harmoniser à un mode de vie. Plutôt que de parler de l'adaptation d'un projet à une société locale, il serait plus juste, en matière d'urbanisme, d'évoquer les répercussions d'un projet sur le comportement humain. Du point de vue de l'hygiène mentale, le zonage - ou la séparation des fonctions à n'importe quelle échelle - est considéré comme un facteur de monotonie, d'ennui, d'asthénie (F. Choay, 1965, p. 67).

Pour en finir avec la question de l'adaptation des modèles, il convient de revenir à l'exemple de l'aménagement de Port-Saïd qui illustre comment l'on passe d'un modèle théorique, conçu en coopération, à sa réinteiprétation lors de la phase de conception en détail par les équipes égyptiennes.

De l'urbanisme théorique à l'urbanisme opérationnel

D'une manière générale, le rôle des modèles dans les schémas directeurs est de présenter aux décideurs une gamme de possibilités destinées à atteindre les objectifs prévus. Le plus souvent, parce qu'ils correspondent à une phase de conception préparatoire, ils ne seront pas réalisés. Dans le cas de Port-Saïd, les plans détaillés destinésà être exécutés furent établis, après la phase de planification, par le Bureau arabe d'architecture et de conseil technique (désormais: BAACT) chargé de la conception détaillée de la reconstruction et du développement et rattaché au ministère.

Figure 4 : Plan détaillé d'un îlot de Port-Saïd réalisé par le Bureau arabe d'architecture et de conseil technique

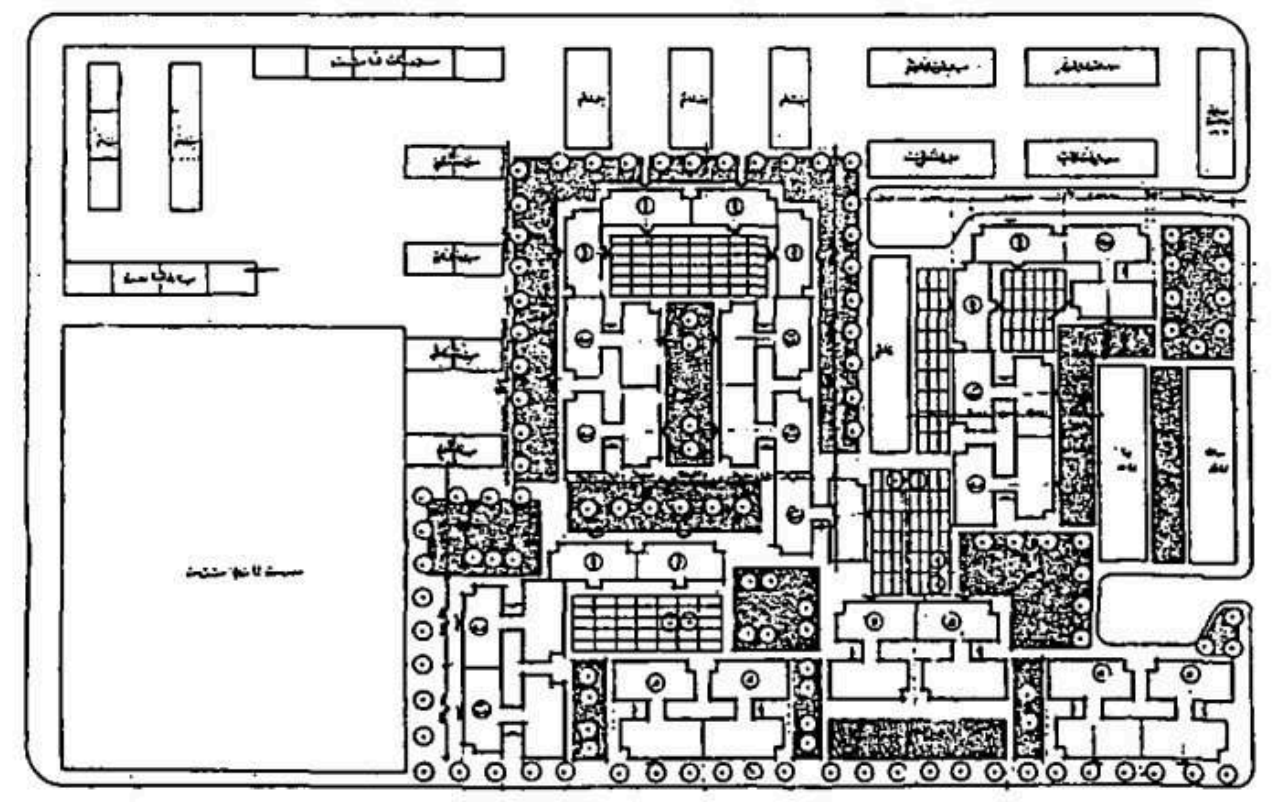

En ce qui concerne le lotissement de parcelles, on peut observer un décalage entre l'urbanisme théorique qui figure dans les plans types proposés dans le schéma directeur, et l'urbanisme finalisé pour un espace précis et conçu par le BAACT qui doit, à l'approche de la mise en œuvre, traduire et insérer l'urbanisme théorique dans la réalité et dans le tissu existant. Les plans détaillés qui décideront du type de construction des nouveaux quartiers sont réalisés par cette organisation, qui représente le plus important groupement d'urbanistes et d'aménageurs égyptiens. 
plans sont élaborés selon des conceptions du quartier qui diffèrent de celles traduites dans les grandes lignes du schéma directeur (cf. figure $4:$ plan détaillé d'un îlot de Port-Saïd réalisé par le BAACT\}. Cette fois, la hiérarchie des rues est moins marquée, les îlots ne sont plus découpés suivant des transversales qui se croisent en formant un quadrillage régulier. Les passages piétonniers sont privilégiés, les voies de circulation secondaires restent en marge du cœur de l'îlot... On peut déceler ici l'adaptation d'un modèle universel de plan type à un style de vie particulier.

Le BAACT est en prise avec la réalité : à ce niveau d'exécution, il est nécessaire de tenir compte du bâti existant puisque la priorité est accordée à la reconstruction. L'urbanisme et l'architecture doivent s'inscrire dans la trame existante lorsque c'est possible :

«Le BAACT chargé de mettre en œuvre les projets a élaboré des plans précis de quartiers ou d'îlots à construire et à reconstruire qui devaient être insérés dans un plan quadrillé, celui qui existait dans la ville ancienne et qui devait orienter la nouvelle croissance urbaine. Nous avons opté pour la réhabilitation en respectant le plan quadrillé de Port-Saïd conçu à l'origine par la Compagnie universelle du canal de Suez pour loger ses ouvriers et employés. Nous avons suivi cette organisation et nous avons aussi essayé de tenir compte du style de bâtiments à arcades que l'on trouve dans les quartiers anciens de Port-Saïd. Quelques types de cet élément architectural ont été reconstruits par le bureau arabe pour garder à ces quartiers un peu de caractère quand la continuité s'imposait. Dans les nouveaux quartiers, c'était impossible car ce type de construction coûte cher. »

Pour des raisons de rentabilité et d'efficacité - la quantité de logements à construire est énorme -, les nouveaux terrains ne seront pas lotis selon cette trame quadrillée et le style HLM remplacera les bâtiments à arcades. Quant à l'architecture, dont on peut penser qu'elle constitue aussi le caractère de la ville, seul l'Organisme du canal de Suez semble la considérer comme un patrimoine de Port-Sai'd; on en fait d'ailleurs un emblème en remettanten état certains des bâtiments qui étaient attribués aux employés du canal. Cette intervention ponctuelle n'empêche pas le paysage urbain de se modifier peu à peu : les immeubles anciens sont détruits au profit de constructions plus neutres qui coûtent moins cher et rapportent davantage en valeur locative.

«Les propriétaires actuels ne sont pas les constructeurs de ces immeubles mais les héritiers. Si, parmi eux, certains sont conscients de la valeur architecturale de leur bâtiment, ils n'ont pas les moyens de l'entretenir. Le gouvernement et les autorités locales ne les y encouragent pas: il n'y a aucune incitation ou prêt et il est impossible d'augmenter les loyers. La loi de blocage des loyers a des conséquences très néfastes sur la survie des immeubles. Par exempte, pour un appartement dans un des immeubles les plus luxueux du centre-ville du Caire, le loyer ne dépasse pas le prix d'un sandwich dans un restaurant populaire. Les gens n'ont aucun intérêt à entretenir leur immeuble puisqu'ils ne gagnent absolument rien. Alors ces témoins de notre patrimoine architectural disparaissent dans toutes les villes. Notre comité d'architectes a toujours dénoncé ces problèmes, mais personne ne nous entend. Et si les hauts responsables sont conscients de l'importance de ces questions, ils disent avoir d'autres priorités. A Port-Saïd, c'est aussi un problème économique: les autorités locales ne proposent aucun encouragement et on ne peut pas demander aux propriétaires de prendre en charge cette réhabilitation. »

En 1973, la question architecturale ne s'est pas davantage posée dans le schéma directeur que dans les projets pris en charge par le BAACT. On peut s'interroger sur la manière dont est perçu le patrimoine attribué à Port-Saïd. Est-il considéré comme un élément de l'identité de la ville? Sa préservation a-t-elle un sens, s'agit-il d'un luxe superflu ? Le bureau arabe chargé de la mise en œuvre avait d'autres priorités liées aux

Égypte/Monde arabe, 23 | 1995 
réalités du moment. Avec l'urgence de la reconstruction, il fallait passer de la théorie, de l'image idéale du schéma directeur, et des méthodes plus ou moins efficaces qu'il préconisait, aux réalisations concrètes, et engager tout le processus qui mènera à la déconstruction du rêve.

Face à la reconstruction : action en urgence et abandon d'un rêve

De manière générale dans les processus d'aménagement, la conception des projets ne représente que la première phase d'un processus plus complexe au cours duquel la réalité remet souvent en cause les rêves énoncés. Soumis, d'une part, à la mise en œuvre effective du projet et à l'intervention des différents acteurs qui en sont chargés et, d'autre part, aux décisions émanant de chaque service administratif disposant d'un pouvoir, le projet initial voit ses objectifs considérablement modifiés. En période d'après-guerre, ce phénomène est d'autant plus flagrant que les conditions réelles sont extrêmes. La phase de reconstruction influence le déroulement du processus d'aménagement, et des enjeux qui dépassent les aménageurs orientent le projet dans d'autres directions. Par ailleurs, si la situation d'après-guerre permet de mobiliser les énergies, elle implique aussi que l'on accorde la priorité aux reconstructions avant d'appliquer le schéma directeur. Les premières révisions des objectifs du schéma directeur s'imposent alors.

Réalités de la mise en œuvre et période de reconstruction

Comme partout en pareille occasion, la situation d'après-guerre dicte les priorités. Le temps est venu d'en finir avec l'abstraction des premiers projets, mais les plans plus précis reprennent en quelque sorte les idées d'organisation sociale et spatiale aperçues dans le schéma directeur :

"Quand les habitants ont été rapatriés, on savait exactement ce qu'on allait réaliser. Les chiffres étaient définitifs, ce n'étaient pas des études approximatives, théoriques ou abstraites. Sur nos plans figuraient les voies de circulation, les lotissements et le type d'habitat qui avait été étudié en fonction des types de familles par quartier. Le bureau arabe d'exécution s'était basé sur les études réalisées par les représentants du ministère des Affaires sociales pour définir le standing des bâtiments et des logements à construire selon le statut de la population. L'enquête du ministère avait identifié les anciens habitants parmi les immigrants, afin d'écarter les imposteurs venus réclamer des logements qu'ils n'avaient jamais habités - et notamment, parmi eux, des habitants des bidonvilles de la périphérie - et proposer des logements adaptés. De cette manière, la reconstruction était très fonctionnelle, tant du point de vue de technique que du point de vue des besoins des familles. Les bâtiments étaient adaptés à leur catégorie socio-professionnelle. On a aussi veillé à mettre en place des services adéquats (enseignement, santé...) pour chaque quartier car à cette époque, rien ne subsistait, il fallait implanter des mosquées, des écoles... »

81 La reconstruction intervient donc dans un souci de retour à l'ordre ancien. Les conditions réelles imposent que l'on s'éloigne en partie des projets d'aménagement. Toutefois, le souci de retrouver tant bien que mal la composition sociale de chaque quartier s'inscrit dans la même volonté de transformer un projet urbain en projet de société, et l'on retrouve à un autre niveau l'utopie sociale dont le schéma directeur s'était fait l'écho. Il s'agit également de mettre chaque catégorie sociale "à sa place ", selon une sorte de ségrégation organisée dans la ville.

«Nous étions contraints d'opter pour une réhabilitation simple et rapide, car au moment où nous étions en train de formuler toutes sortes d'idées dans les rapports commandités, c'était vraiment la bataille de la reconstruction. Nous étions poussés par les autorités gouvernementales qui attendaient des propositions pratiques. » 
82 directeur de Port-Saïd se heurte à l'urgence. Il faut s'éloigner des projets du schéma
directeur pour reconstruire.

«Au fond, nous savions que ces idées de développement étaient plus fantaisistes que réalistes, que tout cela était du temps perdu puisque ces idées étaient irréalisables et qu'il y avait urgence. La reconstruction était en œuvre depuis le premier jour, bien avant que nous nous soyons mis à la recherche de stratégies spatiales. Plus d'un demi million d'habitants étaient dispersés et pesaient comme un fardeau sur le gouvernement égyptien. Reconstruire était prioritaire et cela a été assez rapide. Les ouvriers travaillaient jour et nuit : en trois mois, près de 20.000 habitations furent réparées. Au moment où l'on établissait les plans, les ouvriers étaient déjà sur le chantier, et de temps en temps, on allait les rejoindre avec les plans qui sortaient tout juste de l'atelier. (...) Des plans types permettaient d'amorcer les travaux de fondation alors que les plans définitifs n'étaient pas tout à fait achevés, mais on s'est vite aperçu qu'ils étaient inadaptés à certains lieux. Sur des sols impropres à la construction, il fallait chercher de nouveaux systèmes de fondations. »

On expérimente alors à Port-Saïd la nouvelle technique de l'industrialisation du bâtiment, phase qu'ont connue différents pays en période d'après-guerre. Dans ces conditions, des changements d'options s'avéraient inéluctables.

\section{Changements d'options}

Le bureau arabe de reconstruction réalise vite la difficulté de poursuivre les objectifs initiaux, trop coûteux et trop longs à mettre en place :

« Lorsque nous avons commencé à étudier précisément le budget et à envisager les étapes successives de l'exécution, tout cela a pris des dimensions réelles. Les options du schéma directeur nous ont alors paru trop optimistes, voire fantaisistes. Nous avons dû abandonner nos rêves et devenir réalistes. »

Ainsi, l'idée de déplacer le port de son emplacement au débouché du canal vers le sud, à mi-chemin entre Isma'ilia et Port-Sai'd, de manière à avoir un port très important qui pourrait alimenter l'intérieur du pays et auquel on aurait accès soit par Port-Saïd, soit par Ismaïlia, a été abandonnée « car elle exigeait un budget astronomique ». De même en ce qui concerne le triplement de surface de la ville par l'extension sur les terres du lac Manzala :

«Il y avait plus urgent et c'est seulement en 1981 que l'on a commencé à parler sérieusement de remblayer des terres. Nous nous sommes alors rendu compte qu'on ne pouvait pas faire un nouveau Port-Saïd et les options ont complètement changé. Par exemple, on a renoncé partiellement à miser sur les entrepôts et les zones franches lorsqu'on a constaté le peu d'enthousiasme des investisseurs étrangers. Aujourd'hui, la zone franche n'a toujours pas un succès extraordinaire. » Opérateurs de la reconstruction : un lobby influent

Les documents d'aménagement sont soumis par la suite à l'intervention de toute une série d'acteurs. Grâce à une marge de manœuvre plus ou moins grande, ils ont la possibilité de remanier voire de réorienter le cours des projets: c'est le cas des techniciens chargés de l'exécution puisque la réalisation de plans à une échelle fine leur appartient, mais aussi celui des promoteurs :

«Une organisation gouvernementale avait été constituée pour reconstruire toute la zone du canal de Suez, sous la direction de l'ingénieur Kenawi, ancien responsable du projet du haut barrage d'Assouan. Elle mobilisait les plus grandes firmes de construction en Égypte: 'Uthman Ahmad 'Uthman avec Arab Contractors, Hassan Allam, Misr al-Asmant al-Mussallah (la Compagnie du béton armé égyptienne) et

Égypte/Monde arabe, 23 | 1995 
disposait d'un pouvoir de décision très important... Les plus grandes entreprises d'Égypte travaillaient uniquement pour le développement de la zone du canal sous l'égide de la General Organization for Development and Reconstruction du ministère. Le chantier de Port-Saïd avait été attribué à Arab Contractors, d'autres sociétés s'occupaient d'Ismailia et de Suez. »

87 A la fin de 1973, 'Uthman Ahmad 'Uthman, ministre de la Reconstruction, demeurait le personnage important de l'entreprise Arab Contractors bien qu'il ait cessé d'en être le directeur officiel après sa nationalisation. Il était en mesure d'orienter la reconstruction selon les intérêts de l'entreprise, au moins dans le cas de Port-Saïd où Arab Contractors s'était vu attribuer l'exclusivité des travaux :

« A cette époque. 'U. A. 'Uthman n'avait rien à faire avec Arab Contractors. Il devait s'en dégager complètement pour être ministre. Toutes ces sociétés appartenaient au secteur public, il n'y avait ni gain ni perte parce que les prix étaient fixés par le gouvernement. Il ne s'agissait pas de faire des affaires, mais plutôt de provoquer une grande mobilisation nationale. »

Toutefois, 'Uthman Ahmad 'Uthman n'est pas resté ministre plus de trois ans et son mandat a alimenté les polémiques concernant son engagement dans la reconstruction du canal.

«Il est resté ministre suffisamment longtemps pour pousser le travail de reconstruction. C'était le seul personnage qui pouvait mener cette opération de manière dynamique et rapide car il n'avait pas la mentalité d'un fonctionnaire ou d'un bureaucrate mais celle d'un entrepreneur ; c'est la raison pour laquelle il avait été choisi à l'époque. Pour manipuler de telles sommes et des travaux d'une telle envergure, il faut avoir un esprit très libre. Par exemple, il donnait des primes, pour les encourager, à des ouvriers qui touchaient déjà un salaire relativement élevé. On lui a d'ailleurs reproché par la suite d'avoir gaspillé de l'argent: les prix pouvaient monter puisqu'il n'y avait pas de concurrence et que les projets étaient alloués à ces entreprises sans que les conditions financières soit fixées au préalable. Ils pouvaient donc arguer de la difficulté d'un terrain 'miné' pour pratiquer des tarifs plus élevés qu'en temps normal. »

Dans L'automne de la colère (1983), Muhammad Hasanayn HaykaI rapporte qu'en 1976, 'Uthman Ahmad 'Uthman démissionne de son poste de ministre suite à des «harcèlements en tant que personnage officiel». II explique aussi qu'ayant dû se retirer de Arab Contractors pour accéder au poste de ministre, il « consentit » à devenir président honoraire de la Compagnie et nomma son frère Husayn 'Uthman président de la société. Le temps manquait pour mettre les travaux en adjudication, et le ministre de la Reconstruction dut répartir les travaux à effectuer sans passer par cette formalité. Ce ministre étant 'Uthman Ahmad 'Uthman, il occupait la position idéale pour assurer des contrats à ses sociétés et décider de leurs modalités de paiement. Par la suite, des questions furent posées au parlement sur la qualité douteuse d'une grande partie des bâtiments, du projet de tunnel sous le canal de Suez et de la mise en valeur des terres de Salhiyia.

On pourrait émettre l'hypothèse que les acteurs de la construction ont aussi la capacité de dévier en partie le projet, par exemple en négligeant la qualité au profit de gains matériels immédiats. Dans le cas de Port-Saïd, Yahya al-Zayni nous assure que «les constructions étaient satisfaisantes, aussi bien du point de vue technique que de leur adaptation à la population". Mais d'autres obstacles allaient se dresser entre le rêve des planificateurs et la réalité.

Les limites du pouvoir décisionnaire des aménageurs 
91 Élaborer des documents d'aménagement incombe à un organisme d'État relativement autonome lié à des experts étrangers, et les projets sont soumis à divers acteurs qui interviennent parallèlement ou postérieurement à la phase de planification. Toutefois, la prise de décision appartient en priorité à l'État central.

En amont : les enjeux nationaux et internationaux qui influent sur la prise de décision Yahya al-Zayni est sensible à la manière dont, dans la sphère des décideurs, la reconstruction peut constituer un enjeu national et international, voire commercial. Il met en avant les blocages liés à la répartition des pouvoirs de décision :

«Les documents préparatoires et schémas directeurs sont des documents de travail, des idées sont soumises aux décideurs: le gouverneur ou le ministre concerné, ou encore la présidence. Ils voient exactement où chaque élément du schéma directeur se situe dans le budget, dans les stratégies du pays, dans les priorités... »

93 En amont, la volonté étatique oriente les propositions des schémas directeurs. C'est ainsi qu'ont été développées les liaisons en direction du Sinaï (tunnels, routes, ponts en projet pour traverser le canal de Suez). En 1973, il s'agissait de récupérer les terres occupées du Sinaï; désormais, il est question de créer les conditions permettant de garder une place de choix dans la région en fonction des recompositions du MoyenOrient.

Dès lors qu'il s'agit d'enjeux politiques, ceux-ci se mêlent à des intérêts économiques. Quand, par exemple, les États-Unis prennent position dans la région, c'est non seulement pour assurer la paix, mais aussi la maîtrise du canal de Suez, et enfin parce qu'il y a des marchés à la clé. Parallèlement, le gouvernement égyptien a besoin d'attirer les financements et investissements arabes et occidentaux (japonais par la suite), et toute la population requise pour faire poids autour du canal et désengorger la vallée du Nil. L'ambition des schémas directeurs, qui paraissait manquer de réalisme, a peut-être une autre fonction: elle serait chargée de séduire les décideurs et investisseurs :

«Le ministre de fa Reconstruction appréciait cette ambition lorsque nous lui avons présenté le schéma directeur. On doit d'abord voir les choses en grand, c'est comme cela dans le monde entier. Et finalement, ce sont les stratégies nationales qui orientent la décision. »

En aval : les décisions au coup par coup créent d'autres blocages

La volonté étatique est relativement fragmentée : elle se partage entre des hommes au pouvoir et aux intérêts divergents, dont certains disposent d'appuis leur permettant de faire pencher les décisions du côté opposé aux prévisions des planificateurs. Ce sont des décisions individuelles, au coup par coup, qui sont du même ordre que les décisions nationales stratégiques mais à une autre échelle.

Ainsi Damiette, sur la rive méditerranéenne du Delta à une centaine de kilomètres à l'est de Port-Saïd, s'est vu attribuer un nouveau complexe portuaire agrémenté d'une zone franche et d'une ville nouvelle. Il peut paraître contradictoire d'avoir reporté les investissements portuaires sur cette ville au moment où il était prévu de développer la fonction d'approvisionnement de Port-Saïd, et alors même que dernier n'était pas parfaitement aménagé et ne dispose toujours pas de liaison aisée avec le Delta et le reste de l'Égypte :

«Il était assez maladroit de développer Damiette avant que Port-Saïd n'ait pris tout son élan. Un litige a d'ailleurs opposé les deux ports, chacun essayant d'attirer à soi les lignes directes des compagnies maritimes. L'affaire est allée jusqu'au parlement 
et le ministre du Développement et de la Reconstruction de l'époque a été accusé, parce qu'il était originaire de Damiette, de vouloir pousser sa ville en avant. » pressions politiques contribuent à l'abandon de certains projets au profit d'autres, au gré des jeux d'influence. Les enjeux sont parfois personnels et ne sont pas l'expression d'une revendication du pouvoir par un groupe urbain. Le développement du port de Damiette répond à certains arguments, même s'il signifie un désengagement de PortSaïd :

«Le port de Damiette existait déjà lorsqu'il y a dix ans environ, la proposition de l'agrandir considérablement a été retenue. Il s'agissait de spécialiser Damiette dans le transport de conteneurs, qui ne pouvait se faire à Port-Saïd compte-tenu de la surface disponible. Malgré cela, je crois que c'était un peu prématuré. Mais ce n'est qu'un exemple des changements de priorités qui ont affecté la politique d'aménagement égyptienne. »

Les réorientations de cette politique sont liées à des actions au coup par coup. D'abord pour répondre à un problème précis; d'ailleurs, « les grands travaux d'urbanisme ont été le plus souvent accomplis de manière pragmatique, pour résoudre des problèmes précis comme le Paris d'Haussmann. » (J. Lévy, 1994, p. 327) Ensuite, parce que face à la réalité de la mise en œuvre, les objectifs sont revus à la baisse et les priorités changent :

« Si, dans les années soixante-dix, la zone du canal et ses trois villes étaient prioritaires, il n'est plus question actuellement de miser sur Port-Saïd. Des changements de priorités et le développement d'autres régions ont contribué à moins s'intéresser à la zone du canal. On favorise désormais le Sinaï. »

La priorité accordée aujourd'hui au développement du SinaÏ reste dans le cadre d'une " conquête de l'est ». Elle ne s'apparente plus au gain d'un territoire occupé mais vise un développement au niveau macro-régional.

Ce tableau du processus d'aménagement fait la part belle à l'interventionnisme de l'État, laissant supposer que les acteurs privés locaux n'ont eu qu'à le subir. Si, localement, des détournements ont pu avoir lieu de la part des usagers, le développement de Port-Saïd et ses aléas ont bien été dépendants de la volonté étatique et des encouragements ou du désintérêt relatif du pouvoir central depuis une dizaine d'années. Ces influences, mêlées aux multiples facteurs évoqués, ont mené à l'abandon du rêve d'une ville idéale.

Pour un schéma directeur plus réaliste et une redéfinition du rôle de l'aménageur

101 Le processus d'application des objectifs élaborés met à jour les limites d'un tel type d'aménagement, au moins dans la période proposée. Yahya al-Zayni en vient finalement à remettre en cause le principe d'un certain type de schéma directeur. Nous avons tenté d'associer ses remarques à celles qui reviennent habituellement au sujet de ce type d'aménagement, afin d'identifier un nouveau mode de planification et de resituer, dans ce nouveau cadre, la fonction de l'acteur aménageur.

Nous avons vu de quelle manière l'application des schémas directeurs peut être détournée du document original. Deux éléments ayant trait à la prévision sont à prendre en considération.

103 La première remarque concerne les insuffisances du diagnostic préalable et du manque de réalisme dans les prévisions. Le schéma directeur est issu de perspectives de croissance démographique inquiétantes et surtout, surestimées puisque fondées sur la base des taux de croissance des années soixante/ soixante-dix, qui se sont infléchis par 
la suite. De plus, le postulat des schémas directeurs occidentaux, un contexte dynamique de croissance économique illimitée, semble avoir été repris.

Sur l'anticipation et la prospective, nous pouvons nous référer à l'avis de P. Nora, historien cité par J. Lévy (1994), selon lequel cette dernière est nécessaire « à condition d'admettre [qu'elle] ne sert pas à prévoir l'avenir... Ceci n'est pas un paradoxe: la prospective consiste en fait à prendre une meilleure conscience du présent (...). A partir de là, l'important est de dégager un accord non pas sur un avenir mais sur un horizon. »

Un second thème, lié aux problèmes de la prévision, remet en cause la conception même de la ville et l'action que l'on peut avoir à ce sujet telles qu'elles apparaissent dans les documents d'aménagement :

"Le problème se pose actuellement en science de l'urbanisme et dans les théories du développement: il ne reste plus d'idées de schémas directeurs. Ce sont des objets théoriques auxquels échappent seulement l'aménagement du territoire et la réhabilitation. Mais il est inutile de faire un schéma directeur en espérant qu'il sera suivi, c'est une orientation générale qui permet de prendre des libertés. L'accroissement d'une ville est quelque chose d'organique: elle grandit, elle acquiert des comportements comme les êtres humains, et on ne peut pas prévoir comment, et à quelle cadence exacte, les gens vont agir. On ne peut pas tout prévoir et c'est pourquoi la planification de la croissance est, selon moi, une opération dynamique : elle doit sans cesse évoluer en fonction de l'état du moment »

Yahya al-Zayni cherche dans la réalité le fondement de l'aménagement urbain ; il ne préconise pas la technique prévisionnelle comme fondement de l'action urbaine.

En France et en Grande-Bretagne, un vaste courant critique a abouti, dans les années soixante-dix, à des propositions pour un changement méthodologique dans la planification : il visait à identifier les ressources, mobiliser les moyens plutôt que partir des objectifs. En Occident, des leçons on été tirées des schémas directeurs de la génération 1965 (C. Chaline, 1989) qui s'appliqueraient aussi à l'aménagement de PortSaïd :

- prendre plus en compte les multiples inerties, blocages, conflits d'intérêts, qui rendent aléatoires la mise en œuvre des propositions d'un schéma directeur ;

- substituer des attitudes plus pragmatiques aux comportements volontaristes, identifier les conditions d'une planification plus efficace et plus proche des réalités constatées.

Une question demeure: comment transposer ces leçons? Le recours aux experts étrangers avait déjà eu des effets pervers qui ont parfois abouti à transformer les ingénieurs du pays en adjoints techniques des ingénieurs étrangers. E. Longuenesse (1990) montre comment les espoirs de modernisation provoquent dans un premier temps l'accroissement de la dépendance technologique. Mais ce qui est le cas lorsqu'on parle des ingénieurs et du développement industriel ne s'applique pas aussi directement à l'aménagement du canal. Il y a, d'une part, l'exception d'Arab Contractors, entreprise de construction purement égyptienne et déjà puissante, et d'autre-part, la compétence des experts égyptiens. D'après Yahya al-Zayni, les équipes égyptiennes étaient tout autant capables d'élaborer des schémas directeurs que les experts étrangers, et elles ont seulement bénéficié d'un soutien financier assorti d'une mobilisation internationale associée au retour rapide de la paix dans la zone du canal de Suez.

On peut, avec C. Chaline (1989), considérer que l'utilisation, dans les pays en voie de développement, d'outils tels que les schémas directeurs dans les années soixante-cinq/ 
soixante-quinze pouvait présenter un maximum de bénéfices si le transfert était assorti d'un certain nombre de précautions. Avec un outil, on transférait aussi un modèle qui devait être adapté à la spécificité des conditions sociales, économiques et politiques nationales, notamment à l'insuffisance de gestionnaires chargés de la mise en œuvre au niveau local, et à la faiblesse des ressources financières. Or, dans le cas du schéma directeur de Port-Saïd, le contexte de reconstruction et l'implication du Programme des Nations Unies pour le développement offraient des opportunités de financement pour la conception des documents d'aménagement. L'ambiance du moment laissait donc espérer que les ressources nécessaires à la réalisation des rêves les plus ambitieux seraient disponibles.

110 Par ailleurs, les conditions sont réunies depuis 1992, date de l'élaboration du nouveau schéma directeur de Port-Saïd par une équipe entièrement égyptienne, pour que les aménageurs égyptiens "mettent en œuvre les capacités d'innovation, d'invention, d'initiatives nécessaires pour prendre la place des experts étrangers » (E. Longuenesse; p. 19, 1990).

Les objectifs du projet de 1992 ont évolué vers un développement urbain, économique et social équilibré, suivant la tendance des schémas directeurs de la deuxième génération (C. Chaline, 1989), mais font toujours la part belle au grand projet d'extension de la ville sur un arrière-pays rural en construction, et sa transformation en un centre urbain régional assorti de villes satellites. La démarche est sensiblement la même : on détermine les potentiels liés aux conditions naturelles et, étrangement, l'accent est mis sur la diversification des activités en favorisant un secteur agricole jusqu'alors pratiquement inexistant et destiné à se développer sur les eaux marécageuses peu à peu comblées. Ces nouvelles options sont-elles plus proches de la réalité et les aménageurs égyptiens jouent-ils désormais un rôle moteur dans le développement? Elles ne semblent pas se rattacher au nouveau schéma théorique de la planification urbaine qui émerge actuellement, si ce n'est en ce qui concerne l'actualisation des objectifs: évaluation des besoins socialement différenciés, évaluation des ressources disponibles, propositions axées sur quelques projets structurants, observation continue et actualisation des objectifs.

112 Selon Yahya al-Zayni, le schéma directeur devrait être avant tout une opération dynamique :

«Il faudrait toujours revoir le schéma directeur, l'actualiser, le corriger en fonction des premiers résultats et de leurs effets, en fonction du rythme de développement. La comparaison entre le développement spontané et les idées préconçues du document d'aménagement est nécessaire à tout moment pour réorienter les projets vers plus de réalisme. L'évolution visible doit permettre de corriger les plans et de mettre en place les projets selon le mouvement du développement naturel. »

113 II revient sur la nécessité d'un suivi et de réajustements successifs qui devenaient pratique courante en Europe au moment où fut conçu l'aménagement de Port-Saïd après la guerre. Les schémas directeurs sont là pour fournir un cadre d'action, des objectifs, afin de pouvoir diriger l'action au jour le jour :

«Mais un cadre d'action peut aussi constituer une limitation étouffante. Je dirais plutôt que le schéma directeur indique des orientations générales. Par exemple, entre ce qui était proposé il y a vingt ans pour ce qu'on appelait le «long term strategy » et aujourd'hui, tout a changé. »

114 La fonction principale du schéma directeur serait peut-être de présenter un «appel d'offre » satisfaisant à la fois pour les décideurs et pour les futurs investisseurs. Les 
architectes-aménageurs s'en remettent finalement à l'État. Leur rôle n'est pas tant de s'inscrire dans la réalité que de proposer une gamme variée de projets parmi lesquels seront retenus ceux qui correspondent à la politique plus générale du moment. Il n'est donc pas nécessaire de chercher une logique stricte à un processus d'aménagement, entre ce qui est écrit dans les documents d'aménagement et ce qui est réalisé. De même que, si logique il y a dans les schémas directeurs, il y a une certaine probabilité pour qu'elle soit interrompue lors des étapes ultérieures au gré des différents pouvoirs de décision et d'action. Dans cette perspective, faut-il reconsidérer le rôle de l'architecteaménageur? M. Volait montrait, à proposée l'architecture égyptienne, que "par les possibilités d'intervention au sein de l'État, il a pu participer aux grandes orientations du pays : il ne fut pas exclu du politique ». Or, si la responsabilité de l'élaboration du projet urbain incombe à un organisme d'État et à des experts étrangers, si les intervenants sectoriels ont un poids certain, le pouvoir de décision échappe bel et bien aux aménageurs. La décision appartient en dernier ressort au pouvoir central, ce que l'on pourrait considérer comme un ultime pouvoir de contrôle, puis elle peut être déformée lors des étapes de la mise en œuvre.

L'aménageur est cantonné dans un rôle de technicien mais il peut aussi être vu comme un acteur investi d'une mission de conseil technique - bien que celui-ci semble assez limité avant que l'étape de la mise en œuvre n'ait commencé et permis de matérialiser les limites de ce qui est réalisable - de paix, dans le cas précis de Port-Saïd, et d'annonce. Les architectes-aménageurs ne sont pas seulement des détenteurs de savoir et de compétences; dans une certaine mesure, ils peuvent aussi incarner les intermédiaires entre la volonté du pouvoir central et les aspi rations des sociétés locales.

\section{BIBLIOGRAPHIE}

Chaline C., Urbanisme et développement. Évaluation de quatre schémas directeurs métropolitains :

Ankara. Le Caire, Rabat, Tunis, SEDES, Paris, 1989, 167 p.

Choay F., L'urbanisme, utopies et réalités, Seuil, Paris, 1965, 445 p.

David J.-Cl., «Ingénieurs, urbanisme el pouvoirs locaux à Alep », in Bâtisseurs et bureaucrates: ingénieurs et société au Maghreb et au Moyen-Orient, Maison de l'Orient, Études sur le monde arabe n4, Lyon, 1990, p. 281-290.

Haykal M. H., L'automne de la colère, Ramsay, Paris, 1983.

Lévy J., L'espace légitime, Presses de la Fondation nationale des sciences politiques, Paris, 1994, 442 p.

Longuenesse E., «Ingénieurs au Maghreb et au Moyen-Orient, politiques de développement et nouveaux acteurs sociaux », in Bâtisseurs et bureaucrates... (Voir DAVID, op. cit.). p. 9-28.

Lussault M., Tours : images de la ville et politique urbaine, Maison des sciences de la ville. Université François Rabelais, Tours, Coll. Sciences de la ville $n^{\circ} 3,1993.415 p$. 
Pérennes J.-J., « Les ingénieurs et la politique hydro-agricole au Maroc », in Bâtisseurs et bureaucrates... (Voir DAVID, op. cit.)

Ministry of Housing and Reconstruction, Port-Saïd Masterplan Study, Arab Republic of Egypt. december 1975, $286 \mathrm{p}$.

Roncayolo M., La ville et ses territoires, Gallimard, Folio/Essais, Paris, 1990, 278p.

Volait M., Al-lmara et le débat architectural en Égypte dans les années 1940-1960, mémoire de DEA sous la direction de A. Raymond, Aix-en-Provence, Université de Provence, 1986, 125 p.

Welbank M.. Edwards A., « Port-Saïd: Planning for Reconstruction and Development », Third World Planning Review, vol. 3, n², May 1981, p. 143-160.

\section{NOTES}

1. Les propos de Yahia al-Zayni sont cités à chaque étape en italique et entre guillemets. 2. Ce chiffre, qui concerne l'agglomération du Caire (Le Caire et Giza) et Alexandrie, est cité par G. El Kadi dans « Nouvelles tendances de l'urbanisation en Égypte, ruptures ou continuités ", Égypte/Monde arabe n¹,1er trim. 1990.

3. Des matériaux solides étaient dragués depuis le centre du lac jusqu'aux marges sud et ouest de la ville, où ils étaient accumulés pour constituer de nouvelles terres. C'est encore le système qui prévaut aujourd'hui.

4. Qabutti, ancien village de pêcheur, est toujours un quartier de cabanes, qui abritait 42.488 habitants au recensement de 1986, contre 22.934 en 1976.

5. Source : estimation de la Capmas (Central Agency for Public Mobilization and Statistics).

6. Brunet R., Ferres R., Thery H., Les mots de la géographie, dictionnaire critique, Reclus/La Documentation française, coll. Dynamiques du territoire, 1992, $470 \mathrm{p}$.

INDEX

Mots-clés : architecture, canal de Suez, géographie, guerre, Port-Saïd, urbanisme

\section{AUTEUR}

\section{FRÉDÉRIQUE BRUYAS}

Urbama/Orstom 